\title{
KONGRESSKALENDER
}

\section{September 2014}

03.-06.09.2014, Porto, Portugal

13th Conference

International Association for the Treatment of Sexual Offenders (IATSO)

Ort: University of Porto, Portugal

Information: www.iatso.org

10.-13.09.2014, Prag, Tschechien

European Association of Criminology

14th Annual ESC Conference

Information: http://www.esc-eurocrim.org/

16.-19.09.2014, Bergen, Norwegen

3rd Bergen International Conference on Forensic Psychiatry

Treatment and Management of Psychosis

Radisson Blue Royal Hotel, Bergen

Information: www.bergenconference.no

29.09.-01.10.2014, Potsdam-Griebnitzsee

18. Forensisch-Psychiatrisches

Fallseminar

Alkohol- und Drogendelinquenz; Tötungsdelikte; ambulante Therapie

Prof. Dr. H.-L. Kröber

Institut für Forensische Psychiatrie der

Charité

Information: www.forensik-berlin.de

29.09.-03.10.2014, Königslutter

Curriculum Forensische Psychiatrie und

Psychologie

Die psychiatrisch-psychologische Begutachtung im Strafverfahren, Teil 2

Prof. Dr. N. Konrad, Dr. U. Baltzer, Dr. Ch.

Herbig

Information: www.psychiatrie-akademie.de

\section{Oktober 2014}

Oktober 2014, München

29. Herbsttagung für Forensische

Psychiatrie

Prof. N. N.

Abteilung für Forensische Psychiatrie der Universität München

Information: www.forensik-muenchen.de

23.-26.10.2014, Chicago, Illinois (USA)

American Academy of Psychiatry and the

Law

45th Annual Meeting (with APA)

Chicago Marriott Downtown

Information: www.AAPL.org
28.10.-01.11.2014, San Diego, California (USA)

Association for the Treatment of Sexual Abusers (ATSA)

33nd Annual Research and Treatment Conference

Manchester Grand Hyatt San Diego

Information: www.atsa.com/conference

\section{November 2014}

19.-22.11.2014 San Francisco, California (USA)

American Society of Criminology - Annual Meeting

Neglected Crimes, Groups, Causes and Policy Approaches

San Francisco Marriott Marquis

Information: http://www.asc41.com/annualmeeting.htm

\section{März 2015}

04.-06.03.2015, Lippstadt-Eickelborn 30. Eickelborner Fachtagung zu Fragen der Forensischen Psychiatrie

Kontaktadresse: Michaela.Riepe@wkp-lwl. org

Information: www.lwl-forensik-lippstadt.de

\section{April 2015}

20.-22.04.2015, Potsdam-Griebnitzsee

17. Basiskurs Forensische Psychiatrie: Schuldfähigkeit, Maßregel, Prognose

Prof. Dr. H.-L. Kröber

Institut für Forensische Psychiatrie der Charité

Information: www.forensik-berlin.de

\section{Mai 2015}

08.05.2015, Wien, Allgemeines Krankenhaus Wien

9. Wiener Frühjahrstagung für Forensische Psychiatrie

Prof. Dr. Th. Stompe

Information: Yasmin Haunold, www.ce-management.com

15.05.2015, Dresden

20. Dresdner Frühjahrstagung für Forensische Psychiatrie

Dr. Lau, Dr. Lammel, Dr. Sutarski,

Prof. Dr. Bauer

Information: www.forensik-dresden.de
20.-22.05.2015, Zürich, Schweiz

7. Internationales Symposium Forensische Psychiatrie

World Trade Center

Prof. Endrass, Dr. Rossegger, Prof. Urbaniok Information: www.forensiktagung.ch

\section{Juni 2015}

12.06.2015, Berlin-Dahlem

19. Berliner Junitagung für Forensische Psychiatrie und Psychologie

Institut für Forensische Psychiatrie der

Charité

Information: www.forensik-berlin.de

(Veranstaltungen)

16.06.-18.06.2015, Manchester, UK

15th Annual IAFMHS Conference

Forensic Mental Health Services

University of Manchester

Information: www.iafmhs.org

\section{Juli 2015}

12.-17.07.2015, Wien, Österreich

34th Congress of the IALMH

International Academy of Law and Mental Health

Hotel Intercontinental

Information: www.ialmh.org

Ein internationaler kriminologischer Kongresskalender findet sich unter http://www.asc41.com/caw.html

Hinweise für den Kongresskalender bitte an: hans-ludwig.kroeber@charite.de 